\title{
Fluid Bed Drying Method
}

National Cancer Institute

\section{Source}

National Cancer Institute. Fluid Bed Drying Method. NCI Thesaurus. Code C112980.

A process that removes water or volatile solvents using large volumes of hot air to suspend a bed of solids. 